\title{
A Pancoronavirus RT-PCR Assay for Detection of All Known Coronaviruses
}

\author{
Leen Vijgen, Elien Moës, Els Keyaerts, Sandra Li, and Marc Van Ranst
}

\begin{abstract}
The recent discoveries of novel human coronaviruses, including the coronavirus causing SARS, and the previously unrecognized human coronaviruses HCoV-NL63 and HCoVHKU1, indicate that the family Coronaviridae harbors more members than was previously assumed. All human coronaviruses characterized at present are associated with respiratory illnesses, ranging from mild common colds to more severe lower respiratory tract infections. Since the etiology of a relatively large percentage of respiratory tract diseases remains unidentified, it is possible that for a certain number of these illnesses, a yet unknown viral causative agent may be found. Screening for the presence of novel coronaviruses requires the use of a method that can detect all coronaviruses known at present. In this chapter, we describe a pancoronavirus degenerate primer-based method that allows the detection of all known and possibly unknown coronaviruses by RT-PCR amplification and sequencing of a 251-bp fragment of the coronavirus polymerase gene.
\end{abstract}

Key words: coronavirus; pancoronavirus RT-PCR; degenerate primers; sequencing; polymerase gene

\section{Introduction}

At present, viral culture is the "gold standard" for laboratory diagnosis of respiratory infections. Since coronaviruses are very difficult to grow in cell culture, accurate and sensitive diagnoses are not feasible by this technique. To overcome the lack of sensitivity and to obtain rapid diagnostic results, more sensitive molecular methods for the detection of human coronaviruses (HCoVs) have been developed, including reverse-transcriptase polymerase chain reaction

From: Methods in Molecular Biology, vol. 454: SARS- and Other Coronaviruses,

Edited by: D. Cavanagh, DOI: 10.1007/978-1-59745-181-9_1, C Humana Press, New York, NY 
(RT-PCR), nested RT-PCR, and recently real-time RT-PCR (1). Nevertheless, HCoVs are not often diagnosed in clinical laboratories, although the SARS epidemic and the identification of HCoV-NL63 drew attention to the clinical relevance of HCoVs. Multiplex RT-PCRs for the detection of common respiratory viruses in clinical specimens have now been supplemented with primer pairs for amplification of HCoV-OC43, 229E, and NL63 $(2,3)$.

In order to allow the detection of all known coronaviruses in one assay, consensus RT-PCRs have been developed, based on an alignment of conserved genome regions of several coronaviruses (4). However, the recent identification of HCoV-NL63 demonstrated that this novel coronavirus could not be amplified by the consensus RT-PCR described by Stephensen and colleagues, which might be explained by the presence of several mismatches in the primer sequences. We designed a novel pancoronavirus RT-PCR in which we modified the coronavirus consensus RT-PCR primers based on an alignment of the HCoV-NL63 sequence and the corresponding sequences of 13 other coronaviruses. Theoretically, this pancoronavirus RT-PCR should amplify a 251-bp fragment of the polymerase gene of all coronaviruses known at present, and we tested this experimentally for the five known human coronaviruses (HCoV-NL63, 229E, OC43, HKU1, and SARS-CoV) and three animal coronaviruses (PHEV, FIPV, and MHV). The sensitivity of the pancoronavirus RT-PCR was found to be lower than for a nondegenerate RT-PCR, which can be explained by the high level of degeneracy of the pancoronavirus primers. Nevertheless, this pancoronavirus assay is a useful tool for screening sample collections for the presence of all known and potentially yet unknown coronaviruses (5).

\section{Materials}

\subsection{Sample Handling and RNA Extraction}

1. Viral transport medium (VTM) (BD Diagnostic systems, USA) or phosphate buffered solution (PBS). Store at room temperature.

2. Filter Minisart plus $0.45 \mu \mathrm{m}$ (Sartorius, Vivascience AG).

3. 2-ml syringe without a needle.

4. Glass pearls ( $5 \mathrm{~mm}$ diameter) (Merck MDA, VWR, Belgium).

5. Qiamp viral RNA mini kit (Qiagen, The Netherlands). Store at room temperature, except for the AVL lysis buffer, which should be stored at $4{ }^{\circ} \mathrm{C}$ after addition of carrier RNA.

\subsection{Pancoronavirus One-Step RT-PCR}

1. Primers: Forward primer: Cor-FW (5'-ACWCARHTVAAYYTNAARTAYGC-3') and reverse primer: Cor-RV (5'-TCRCAYTTDGGRTARTCCCA-3') (Eurogentec, Seraing, Belgium). The primers are prepared in a stock solution of $100 \mu \mathrm{M}$. 
A working solution of $15 \mu \mathrm{M}$ is made for use in the RT-PCR. Store at $-20^{\circ} \mathrm{C}$ until use.

2. Qiagen One-Step RT-PCR kit containing One-Step RT-PCR enzyme mix (a combination of Omniscript and Sensiscript reverse transcriptase and HotStarTaq DNA polymerase), One-Step RT-PCR buffer 5X, and dNTP mix, $10 \mathrm{mM}$ each (Qiagen, The Netherlands). Store all reagents at $-20^{\circ} \mathrm{C}$.

3. RNAse-free water (Sigma-Aldrich, Belgium). Store at room temperature.

4. GeneAmp PCR system 9700 thermal cycler (Applied Biosystems, Foster City, CA, USA).

5. 1X Tris-borate EDTA (TBE) buffer: dilute 10X TBE buffer (Invitrogen Life Technologies) 1:10 with MilliQ water (Millipore). Store at room temperature.

6. PAGE gels $\left(6 \%\right.$ acrylamide/ $N, N^{\prime}$-methylenebisacrylamide in $1 \mathrm{X}$ TBE buffer containing $0.064 \%(\mathrm{w} / \mathrm{v})$ ammonium persulfate and $150 \mu \mathrm{l}$ tetramethylethylenediamine). After preparation of a batch of ten gels store at $4^{\circ} \mathrm{C}$.

7. Molecular Weight Marker VI (MWM VI) (Roche, Mannheim, Germany). Store at $4{ }^{\circ} \mathrm{C}$.

8. Imagemaster VDS (Pharmacia, Uppsala, Sweden).

\subsection{Sequencing and Sequence Analysis}

1. MSB Spin PCRapace purification kit (Invitek, Westburg, The Netherlands). Store at room temperature.

2. Saekem GTG agarose for the recovery of nucleic acids (Cambrex, Belgium).

3. 1X Tris acetate EDTA (TAE) buffer: dilute 10X TAE buffer 1:10 with MilliQ water (Millipore). Store at room temperature.

4. QIAquick gel extraction kit (Qiagen, The Netherlands).

5. Forward primer: Cor-FW (5'-ACWCARHTVAAYYTNAARTAYGC-3') or reverse primer Cor-RV (5'-TCRCAYTTDGGRTARTCCCA-3') (Eurogentec, Seraing, Belgium). The primers are prepared in a stock solution of $100 \mu \mathrm{M}$. A working solution of $5 \mu \mathrm{M}$ is made for use in the cycle sequencing reaction. Store at $-20^{\circ} \mathrm{C}$ until use.

6. ABI PRISM BigDye Termination Cycle Sequencing Ready Reaction kit (Applied Biosystems, CA, USA). Store at $-20^{\circ} \mathrm{C}$.

7. Biometra T3000 thermocycler (Biometra, Westburg, The Netherlands).

8. Ethanol absolute.

9. Sodium acetate $3 \mathrm{M} \mathrm{pH}$ 4.6. Store at $4^{\circ} \mathrm{C}$.

10. Formamide. Store at $-20^{\circ} \mathrm{C}$.

11. ABI Prism 3100 Genetic Analyzer (Applied Biosystems, Foster City, CA, USA).

\section{Methods}

\subsection{Sample Handling and RNA Extraction}

1. Nasopharyngeal and throat swabs: place the tip of the swab in a tube containing $3 \mathrm{ml}$ VTM or PBS. After squeezing the tip of the swap and vortexing, take $140 \mu \mathrm{l}$ of the solution for RNA extraction. Store the rest of the sample at $-80^{\circ} \mathrm{C}$. 
2. Bronchoalveolar lavages and nasopharyngeal aspirates: take $140 \mu l$ of the sample for RNA extraction without prior dilution in VTM or PBS. Store the rest of the sample at $-80^{\circ} \mathrm{C}$.

3. Sputum samples: dilute about 100 to $300 \mathrm{mg}$ of sputum in $3 \mathrm{ml}$ VTM or PBS. Add glass pearls and vortex intensively until the solution containing the sputum is completely liquefied. Filter the solution using a 2-ml syringe without a needle and a $0.45-\mu \mathrm{m}$ Minisart Plus filter (see Note 1). Take $140 \mu \mathrm{l}$ of the filtrate for RNA extraction. Store the rest of the sample at $-80^{\circ} \mathrm{C}$.

4. Perform the RNA extraction on $140 \mu$ l of sample according to the manufacturer's instructions using the Spin Protocol (Qiagen QIAamp viral RNA mini kit handbook) (see Note 2$)$. Store the obtained RNA extract $(60 \mu \mathrm{l})$ at $-80^{\circ} \mathrm{C}$.

\subsection{Pancoronavirus One-Step RT-PCR}

1. Screening a sample for the presence of coronaviruses is performed by amplifying a 251-bp fragment of the coronavirus polymerase gene using the following primer set: Cor-FW (5'-ACWCARHTVAAYYTNAARTAYGC-3') and Cor-RV (5'-TCRCAYTTDGGRTA RTCCCA-3') (see Fig. 1) (see Note 3). Prepare the following master mix for RT-PCR according to the number of samples to be tested: $10 \mu 1$ 5X QIAGEN OneStep RT-PCR Buffer, $2 \mu 1$ dNTP mix (final concentration of $400 \mu \mathrm{M}$ of each dNTP), $1.8 \mu$ l QIAGEN OneStep RT-PCR Enzyme Mix, $4 \mu \mathrm{M}$ of forward and reverse primer, and RNase-free water to $40 \mu$ l per reaction (see Notes 4 and 5).

Forward primer region

$\begin{array}{ll}\text { HCOV-NL63 } & : \\ \text { HCOV-229E } & : \\ \text { FIPV } & : \\ \text { TGEV } & : \\ \text { PEDV } & : \\ \text { HCOV-OC4 } & : \\ \text { BCOV } & : \\ \text { PHEV } & : \\ \text { CRCV } & : \\ \text { MHV } & : \\ \text { SDAV } & : \\ \text { SARS-COV } & : \\ \text { IBV } & : \\ \text { TCOV } & :\end{array}$

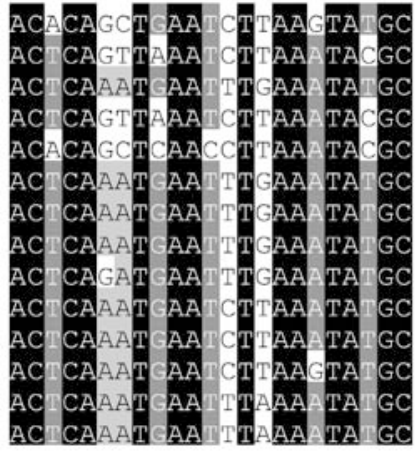

ACWCARHTVAAYYTNAARTAYGC
Reverse primer region

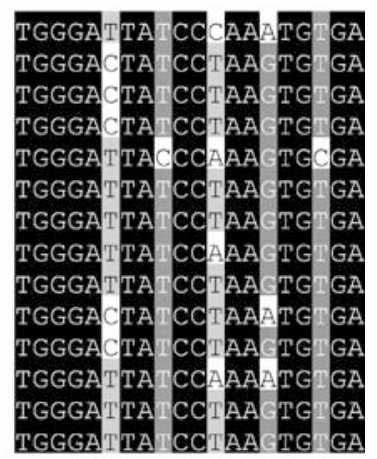

TGGGAYTAYCCHAARTGYGA

Fig. 1. Selection of primers for the novel pancoronavirus RT-PCR. Shown is the alignment of 14 coronaviral sequences of a conserved region of the polymerase gene. The forward (Cor-FW) and reverse (Cor-RV) primer sequences are shown at the bottom ( $\mathrm{Y}=\mathrm{C} / \mathrm{T}, \mathrm{W}=\mathrm{A} / \mathrm{T}, \mathrm{V}=\mathrm{A} / \mathrm{C} / \mathrm{G}, \mathrm{R}=\mathrm{A} / \mathrm{G}, \mathrm{H}=\mathrm{A} / \mathrm{T} / \mathrm{C}, \mathrm{N}=\mathrm{A} / \mathrm{C} / \mathrm{T} / \mathrm{G})$. 
2. Add $10 \mu \mathrm{l}$ RNA-extract to the tube containing $40 \mu \mathrm{l}$ of the master mix, leading to a final reaction volume of $50 \mu \mathrm{l}$ (see Notes 6 and 7). The reaction is carried out with an initial reverse transcription step at $50^{\circ} \mathrm{C}$ for $30 \mathrm{~min}$, followed by PCR activation at $95^{\circ} \mathrm{C}$ for $15 \mathrm{~min}, 50$ cycles of amplification $\left(30 \mathrm{sec}\right.$ at $94^{\circ} \mathrm{C} ; 30 \mathrm{sec}$ at $48^{\circ} \mathrm{C}$; $1 \mathrm{~min}$ at $72^{\circ} \mathrm{C}$ ), and a final extension step at $72^{\circ} \mathrm{C}$ for $10 \mathrm{~min}$ in a GeneAmp PCR system 9700 thermal cycler (Applied Biosystems, Foster City, CA, USA).

3. Load $9 \mu \mathrm{l}$ of PCR product mixed with $1 \mu \mathrm{l}$ loading dye on a $6 \%$ PAGE gel. Load $3 \mu \mathrm{l}$ of MWM VI on each side of the gel (see Note 8). Run the gel at $200 \mathrm{~V}$, $200 \mathrm{~mA}$ for $30 \mathrm{~min}$. Visualize the bands by illuminating the gel with UV-light on an Imagemaster VDS system after staining the gel for 5 min with ethidium bromide (EtBr) (see Note 9).

4. Determination of the fragment size is performed by comparing the length of the bands to MWM VI. Samples from which a PCR product of approximately $251 \mathrm{bp}$ is amplified are presumed to be coronavirus-positive and will be subject to further (sequence) analysis (see Note 10).

\subsection{Sequencing and Sequence Analysis}

1. If a single 251-bp band is visible after EtBr staining, purify the total amplification product by using the MSB Spin PCRapace purification kit according to the manufacturer's instructions (Invitek MSB Spin PCRapace purification kit handbook).

2. If more than one band is visible, including a 251-bp band, run the total amount of PCR product mixed with $5 \mu$ l loading dye on a $1.5 \%$ agarose gel (in $1 \mathrm{X}$ TAE buffer) on $100 \mathrm{~V}, 500 \mathrm{~mA}$ for $1 \mathrm{~h}$. Stain for $10 \mathrm{~min}$ with EtBr, and cut each band from the gel with a sterile scalpel. Purify the fragments excised from the agarose gel using the QIAquick gel extraction kit according to the Spin Protocol in the QIAquick gel extraction kit handbook.

3. For sequencing, add $5 \mu \mathrm{l}$ of purified amplification product to $4 \mu \mathrm{l}$ of ABI PRISM BigDye Ready Reaction mix and $1 \mu \mathrm{l}$ of a $5-\mu \mathrm{M}$ solution of forward or reverse primer. The sequence reaction, which consists of 25 cycles of $30 \mathrm{sec}$ at $96^{\circ} \mathrm{C}, 15$ sec at $50^{\circ} \mathrm{C}$, and $4 \mathrm{~min}$ at $60^{\circ} \mathrm{C}$, is performed in a Biometra T3000 Thermocycler.

4. After cycle sequencing, precipitate the DNA products by adding $62.5 \mu 1$ absolute ethanol, $3 \mu \mathrm{l}$ sodium acetate, and $24.5 \mu \mathrm{l}$ MilliQ water per sample. Mix the samples and leave them at room temperature for $15 \mathrm{~min}$, followed by a centrifugation for $20 \mathrm{~min}$ at 13,000 rpm (see Note 11). Carefully aspirate the supernatant, wash the pellet by adding $150 \mu 170 \%$ ethanol, and centrifuge the samples for $5 \mathrm{~min}$ at $13,000 \mathrm{rpm}$. Carefully aspirate the supernatant, and let the pellet dry for $15 \mathrm{~min}$ at room temperature. Dissolve the pellet in $15 \mu \mathrm{l}$ of formamide and denature for 2 min at $95^{\circ} \mathrm{C}$. The samples are then stored at $-20^{\circ} \mathrm{C}$ before analysis on an ABI Prism 3100 Genetic Analyzer (Applied Biosystems, Foster City, CA, USA).

5. Chromatrogram sequencing files can be inspected with Chromas 2.2 (Technelysium, Helensvale, Australia), and contigs of the fragments sequenced by Cor-FW 
and Cor-RV, respectively, can be prepared using SeqMan II (DNASTAR, Madison, WI). The contig sequence data can easily be arranged into a FASTA format by saving the consensus as a single text file in the Seqman program. The text file will automatically have the following format:

\section{$>$ sequence title (hard return) \\ GACGAGTAATTGC. . . (not containing hard returns)}

Such a FASTA file can then be used for nucleotide similarity searches using the NCBI WWW-BLAST (basic local alignment search tool) server on the GenBank DNA database release 155.0 (6). The coronavirus type that is present in the sample can be determined by using this BLAST analysis, which provides coronavirus sequences that show the highest nucleotide similarity with the sequenced fragment (see Note 12). Furthermore, a FASTA file of the sequence data can be loaded into a multiple sequence alignment program such as CLUSTAL $\mathrm{X}$ version 1.82 (7), and the resulting alignment can be manually edited in the

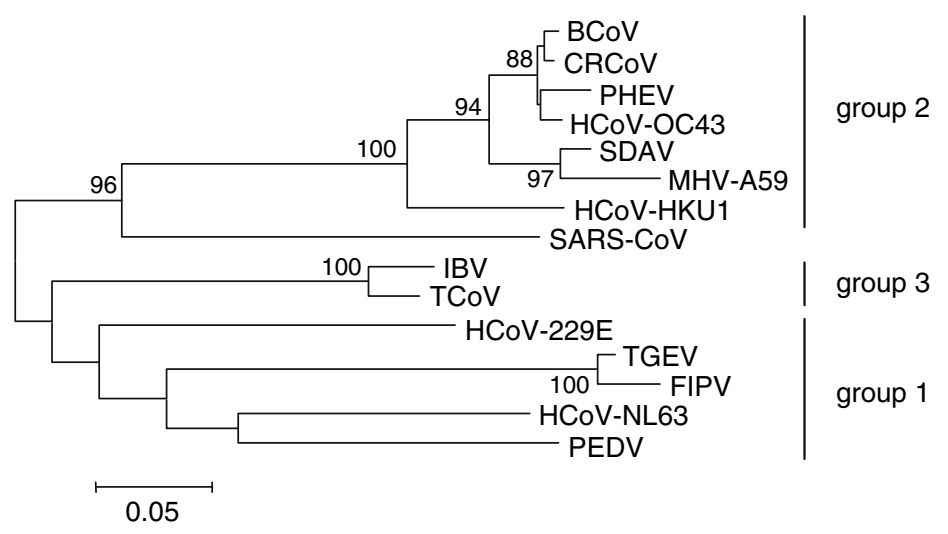

Fig. 2. Neighbor-joining phylogenetic tree of coronavirus partial polymerase gene sequence data (corresponding to the pancoronavirus fragment). The frequency of occurrence of particular bifurcations (percentage of 500 bootstrap replicate calculations) is indicated at the nodes. Bootstrap values over $75 \%$ are shown. The coronavirus sequences used here are available from GenBank under the following accession numbers: HCoV-NL63, AY567487; HCoV-229E, AF304460; PEDV, AF353511; transmissible gastroenteritis virus (TGEV), AF304460; feline infectious peritonitis virus (FIPV), AF124987; HCoV-OC43, AY391777; HCoV-HKU1, NC 006577 ; porcine hemagglutinating encephalomyelitis virus (PHEV), AF124988; bovine coronavirus (BCoV), AF391541; mouse hepatitis virus (MHV-A59), X51939; sialodacryoadenitis virus (SDAV), AF124990; canine respiratory coronavirus (CRCV), AY150273; SARS-CoV, AY313906; turkey coronavirus (TCoV), AF124991; and infectious bronchitis virus (IBV), Z30541. 
GeneDoc Alignment editor (8). Starting from a sequence alignment that compares nucleotides at homologous sites, i.e., sharing a common ancestor, a phylogenetic tree can be constructed using phylogenetic programs such as MEGA version 3.1 (Fig. 2) (9). The construction of a phylogenetic tree based on sequence data of samples and coronavirus sequence data from GenBank will provide information regarding the genetic and evolutionary relatedness of a coronavirus detected in a sample to other known coronaviruses (see Note 13).

\section{Notes}

1. Sputum samples diluted in VTM or PBS should be filtered prior to analysis in order to remove large amounts of cellular debris. It can be difficult to completely liquefy the sputum sample, so the filter may become blocked. In this case, the only solution is to use another filter.

2. Especially during RNA extraction, the likelihood of cross-contamination is high. When performing the RNA extraction manually, one should change tips in between samples, as well as when adding wash buffer to the samples. Another precautionary measure that can be taken is to use tissue paper when opening the samples to avoid the formation of aerosols.

3. For modification of the coronavirus consensus RT-PCR primers (4), an alignment of the polymerase gene sequences of 14 known coronaviruses was made (Fig. 1). Degenerate Cor-FW and Cor-RV primers were developed. The coordinates of Cor-FW and Cor-RV are 14017 and 14248, respectively, in the HCoVNL63 complete genome sequence. The 14 coronavirus sequences used here are available from GenBank under the following accession numbers: $\mathrm{HCoV}$ NL63, AY567487; HCoV-229E, AF304460; PEDV, AF353511; transmissible gastroenteritis virus (TGEV), AF304460; feline infectious peritonitis virus (FIPV), AF124987; HCoV-OC43, AY391777; porcine hemagglutinating encephalomyelitis virus (PHEV), AF124988; bovine coronavirus (BCoV), AF391541; mouse hepatitis virus (MHV-A59), X51939; sialodacryoadenitis virus (SDAV), AF124990; canine respiratory coronavirus (CRCV), AY150273; SARS-CoV, AY313906; turkey coronavirus (TCoV), AF124991; and infectious bronchitis virus (IBV), Z30541.

4. The sensitivity of the pancoronavirus RT-PCR assay was assessed by testing tenfold dilutions of HCoV-NL63 and HCoV-OC43 RNA. Primer concentrations were increased up to $4 \mu \mathrm{M}$ per reaction to improve the sensitivity of the assay. While 50 copies of HCoV-OC43 RNA copies per microliter sample could be detected, the sensitivity for HCoV-NL63 was slightly lower, i.e., $5 \times 10^{3}$ RNA copies per microliter sample.

5. An important precautionary measure when performing RT-PCR is to avoid RNase contamination. Therefore, all materials should be RNase-free, and gloves and a gown should be worn even when preparing the (noninfectious) master mix. In addition, an RNase inhibitor (RNase OUT, Invitrogen Life Technologies; 20 units per reaction) can be added to the RT-PCR reaction to inhibit degradation 
of the RNA sample owing to RNases. This measure should be taken especially when a positive control RNA sample no longer amplifies.

6. Include negative as well as positive controls in each RT-PCR. A negative control consists of a sample containing only the master mix without RNA (10 $\mu$ l of water is added instead of RNA). As a positive control, RNA of a human coronavirus (e.g., HCoV-OC43) can be used.

7. When adding the RNA samples to the master mix, precaution must be taken to avoid RNA contamination. Such a precautionary measure is opening the tubes with tissue paper, thereby avoiding the formation of aerosols, as the latter could lead to cross-sample contamination.

8. It is advised to load the Molecular Weight Marker on both sides of the gel, as a gel can show the phenomenon of "smiling," which makes the determination of the fragment size difficult when the MWM is only present on one side of the gel.

9. Use two pairs of gloves when handling ethidium bromide, as this intercalating agent is highly mutagenic.

10. Amplification of a 251-bp fragment of the coronavirus polymerase gene was demonstrated for HCoV-NL63, HCoV-OC43, HCoV-229E, SARS-CoV, PHEV, FIPV, and MHV-A59 (Fig. 3) and for HCoV-HKU1 (data not shown).

11. The DNA pellet is often not visible. Therefore, during centrifugation the tubes are always put in the same orientation in the centrifuge, so that one knows at which side of the tube the pellet is located. The aspiration of supernatant should be performed carefully without touching the pellet.

12. The polymerase gene is a very conserved region in the coronavirus genome. Therefore, the percentage sequence similarity will be high among different coronavirus members belonging to the same group. Nevertheless, this sequence information allows a primary identification of the coronavirus type that is present in a sample.

VI NL63 OC43 229E SARS PHEV FIPV MHV

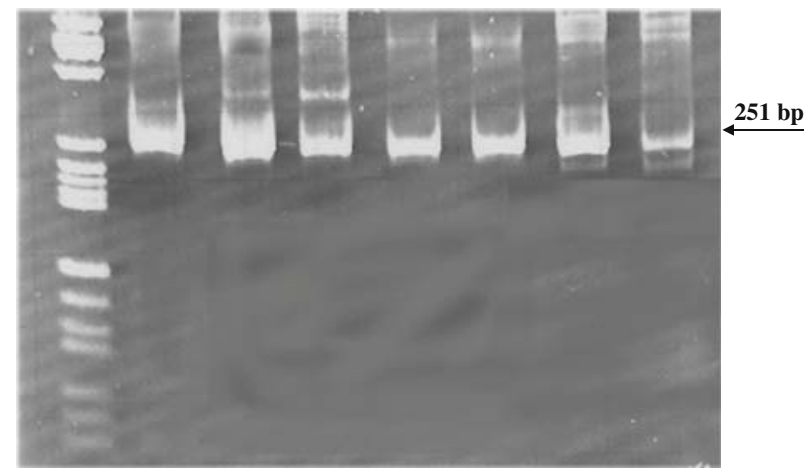

Fig. 3. Gel electrophoresis after pancoronavirus RT-PCR assay. The indicated band of $251 \mathrm{bp}$ corresponds with the expected amplicon size. Molecular Weight Marker VI was used as a marker (Roche, Mannheim, Germany). 
13. The genetic and evolutionary relatedness to known coronaviruses can be determined from the phylogenetic tree clustering pattern of the coronavirus sequence fragment, amplified from a sample. A phylogenetic tree is composed of nodes that are connected by branches. The external nodes represent the existing organisms or taxa, while the internal nodes represent the (hypothetical) ancestors of the existing taxa. The length of the horizontal branches is indicative of the evolutionary distance between the connected nodes, whereas the vertical branches are noninformative. Taxa that are present in the same cluster are monophyletic, which indicates that they show a (relatively) close genetic relatedness.

\section{Acknowledgments}

We would like to thank all the colleagues of the laboratory of Clinical and Epidemiological Virology, Department of Microbiology and Immunology, Rega Institute for Medical Research, University of Leuven, Belgium, and Dr. Lia van der Hoek and Prof. Dr. Ben Berkhout of the Department of Retrovirology, Academic Medical Center, University of Amsterdam, The Netherlands, for helpful comments and discussion. This work was supported by a postdoctoral fellowship (P.D.M.) of the Research Fund K.U. Leuven to Leen Vijgen.

\section{References}

1. van Elden, L. J., van Loon, A. M., van Alphen, F., Hendriksen, K. A., Hoepelman, A. I., van Kraaij, M. G., et al. (2004) Frequent detection of human coronaviruses in clinical specimens from patients with respiratory tract infection by use of a novel real-time reverse-transcriptase polymerase chain reaction. J. Infect. Dis. 189, 652-657.

2. Bellau-Pujol, S., Vabret, A., Legrand, L., Dina, J., Gouarin, S., PetitjeanLecherbonnier, J., et al. (2005) Development of three multiplex RT-PCR assays for the detection of 12 respiratory RNA viruses. J. Virol. Meth. 126, 53-63.

3. Vabret, A., Mourez, T., Dina, J., van der Hoek, L., Gouarin, S., Petitjean, J., et al. (2005) Human coronavirus NL63, France. Emerg. Infect. Dis. 11, 1225-1229.

4. Stephensen, C. B., Casebolt, D. B., and Gangopadhyay, N. N. (1999) Phylogenetic analysis of a highly conserved region of the polymerase gene from 11 coronaviruses and development of a consensus polymerase chain reaction assay. Virus Res. 60, 181-189.

5. Moes, E., Vijgen, L., Keyaerts, E., Zlateva, K., Li, S., Maes, P., et al. (2005) A novel pancoronavirus RT-PCR assay: frequent detection of human coronavirus NL63 in children hospitalized with respiratory tract infections in Belgium. BMC Infect. Dis. 5, 6.

6. Altschul, S. F., Gish, W., Miller, W., Myers, E. W., and Lipman, D. J. (1990) Basic local alignment search tool. J. Mol. Biol. 215, 403-410. 
7. Thompson, J. D., Gibson, T. J., Plewniak, F., Jeanmougin, F., and Higgins, D. G. (1997) The CLUSTAL $X$ windows interface: flexible strategies for multiple sequence alignment aided by quality analysis tools. Nucleic Acids Res. 25, 4876-4882.

8. Nicholas, K. B., Nicholas, H. B., and Deerfield, D. W. (2005) GeneDoc: analysis and visualization of genetic variation. Embet News 4, 14.

9. Kumar, S., Tamura, K., and Nei, M. (2004) MEGA3: integrated software for molecular evolutionary genetics analysis and sequence alignment. Brief Bioinform. 5, 150163. 